\title{
Qualität in der Wikipedia: Binnenperspektive eines Historikers
}

\section{Außenbild: unumgänglich, obwohl jeder mitschreiben darf}

Das Unbehagen, das die Wikipedia vielen Historikern bereitet, scheint sich aus zwei Hauptquellen zu speisen. Zum einen ist die Vorstellung kaum vertrauenerweckend, dass dort ohne Ausnahme jeder des Schreibens auch nur halbwegs Kundige jederzeit Artikel schreiben oder sie verändern darf, und dass es darüber hinaus keine redaktionelle Prüfinstanz ausgebildeter Historiker gibt, keine peer group, kein peer review. Zum anderen ist es alltägliche Erfahrung, dass Studierende gerade beim Einstieg in neue Themen erst einmal die Wikipedia konsultieren. Auf der einen Seite steht also Misstrauen gegenüber Dilettantismus - mit allen Risiken und Nebenwirkung die das selbstverständlich zu begrüßende dilectare zur Folge hat -, auf der anderen Seite die Erfahrung aller Lehrenden, dass die Wikipedia im Studium eine unkontrollierbare Rolle spielt.

Tatsächlich beginnt die Recherche zu einem neuen Thema im Zeitalter des Internets aber meist nicht bei der Wikipedia, sondern man recherchiert mittels der Suchmaschine Google (was einen eigenen Beitrag lohnen würde). Inzwischen erfolgen fast 95 Prozent der Suchanfragen in Deutschland mittels dieser Suchmaschine. ${ }^{1}$ Google präsentiert nach Eingabe eines Suchbegriffs meist zehn Links zu mehr oder minder passenden Seiten im Internet. Die Kriterien, die darüber entscheiden, wer ,ganz oben“ landet, sind dabei Googles Betriebsgeheimnis. Sieht man von gewerblichen Anzeigen ab, so wird unter den ersten drei Treffern - und das ist hier entscheidend - wenn vorhanden immer ein Link auf einen Artikel in der Wikipedia erscheinen. Dies liegt wohl in erster Linie daran, dass die entsprechenden Artikel am häufigsten aufgerufen werden, was wiederum durch die starke Bevorzugung in den Suchmaschinen mitverursacht wird - eine „Empfehlung“ auf Wechselseitigkeit. Schon allein aus diesem Grunde ist die Wikipedia zu einer der wichtigsten Adressen zum Erwerb von Kenntnissen zu einem neuen Thema geworden. Sie belegt in Deutschland bei der Zahl der Aufrufe den siebenten Platz nach Google.de, Facebook.com, Amazon.de, Ebay.de, Youtube.com

1 Laut seo-united.de: http://www.seo-united.de/suchmaschinen.html (27.2.2015).

(@) Br.SA 12015 Hans-Jürgen Hübner, publiziert von De Gruyter.

Dieses Werk ist lizenziert unter der Creative-Commons-Attribution-ShareAlike-3.0-Lizenz. 
und Google.com. ${ }^{2}$ Damit belegt sie unter den nicht-kommerziellen und werbefreien Seiten sogar den ersten Platz. Wer in Deutschland lebt, der erhält auf seine Suchanfrage sogar den Link auf den entsprechenden Artikel der deutschen Sprachversion der Wikipedia.

Dies wirft die Frage auf, warum die Leser, trotz offenkundiger und leicht belegbarer Qualitätsmängel, immer wieder auf diese Seite zurückkehren. Der weit reichende Einfluss der Wikipedia im nicht-kommerziellen Bereich steht letztlich in überraschendem Gegensatz zu der Tatsache, dass diese Enzyklopädie von unbezahlten Freiwilligen erstellt wird - sieht man einmal von lancierten Auftragsarbeiten ab, die allerdings wiederum nur dann akzeptiert werden, wenn sie den in der Wikipedia vereinbarten Grundsätzen entsprechen. Noch offenkundiger werden die Qualitätsprobleme, wenn man sich vor Augen hält, dass jedermann, auch anonym oder unter Pseudonym, mitarbeiten kann, und dass niemand nach seiner Qualifikation gefragt wird.

Doch die Erfahrung mit der Internetenzyklopädie ist eine andere, denn die zentralen Geschichtsartikel haben zu einer beachtlichen Qualität gefunden. Auch entdeckt man dort exzellente Beiträge zu Themenkreisen, über die sich ansonsten nur unter erheblichem Aufwand ein erster Überblick finden lässt. ${ }^{3}$ Zudem darf man den Bildungs- und Ausbildungsstand der „Wikipedianer“ nicht unterschätzen. Allein acht Prozent von ihnen wurden - wenn auch nach eigenen Angaben promoviert, 18 Prozent besitzen einen Mastertitel. ${ }^{4}$

Zum Abfassen von mittlerweile über 1,8 Millionen Artikeln allein in der deutschsprachigen Version der Wikipedia ${ }^{5}$ (zum Vergleich: Brockhaus, 21. Auflage, 2005/06: 300.000 „Stichwörter und erklärte Begriffe“ ${ }^{6}$ können sich doch nicht nur wohlwollende, begeisterte, gut ausgebildete Idealisten versammelt haben, die aus Altruismus ihre Arbeitskraft einbringen. Gibt es darunter keine Menschen, die ganz andere Motive leiten, Schreiberlinge, die Verleumdungskampagnen starten, desinformieren oder provozieren, Eigenwerbung oder von anderen bezahlte Werbung lancieren. Geraten nicht vages Hinterkopfwissen, dazu Geschichtsklitterungen, Beschönigungen, Verharmlosungen oder gar Ewig-Gestriges in die Wi-

2 Laut alexa.com: http://www.alexa.com/topsites/countries/DE (7.3.2015).

3 Die derzeit als ,exzellent“ geltenden Artikel aus dem Bereich der Geschichte finden sich hier: https://de.wikipedia.org/w/index.php?title=Wikipedia:Exzellente_Artikel\&oldid=120540227 (8.3.2015).

4 Vgl. https://meta.wikimedia.org/w/index.php?title=Editor_Survey_2011/Profiles\&oldid=5636718 (8.3.2015).

5 Eine Sammlung von Statistiken zur deutschsprachigen Wikipedia findet sich hier: http://de.wi kipedia.org/wiki/Spezial:Statistik (8.3.2015).

6 Vgl. http://www.brockhaus.de/ (7.3.2015). 
kipedia? Kann dies bei über zwei Millionen registrierten und zahllosen weiteren Nutzern allein in der deutschsprachigen Wikipedia überhaupt verhindert werden?

Nach außen liefert die Wikipedia ein eher ruhiges Bild. Sie tritt als eine Sammlung von Artikeln auf je einer Internetseite auf, die zur Website Wikipedia gehören, im Fall der deutschsprachigen Version unter http://de.wikipedia.org. Diskussionsseiten, die zu jedem Artikel gehören, ermöglichen es den Verfassern der Artikel über ihr Thema ins Gespräch zu kommen. Doch hinter diesem ruhigen Bild verbirgt sich eine höchst vielschichtige, oftmals streitbare, aber auch zunehmend von wissenschaftlichen Grundsätzen bestimmte Tätigkeit, die sich auf der Basis gemeinsam verabschiedeter Grundsätze, Regeln und Entscheidungen entfaltet. Dies ist zugleich die Stelle, an der die Kenntnisse vieler Wissenschaftler über die Wikipedia enden.

\section{Grundsätze: Neutralität, Belegpflicht}

Einer der obersten Grundsätze der größten Enzyklopädie ist die Neutralität oder der „Neutrale Standpunkt“ (,neutral point of view“). Ein Artikel soll dabei alle wesentlichen Standpunkte, Meinungen und Debatten eines Themas beschreiben und charakterisieren, ohne einzelne davon zu befürworten oder abzulehnen. Es soll aufgezeigt werden, wer bis dato welche Meinung in welchen Publikationen vertreten hat, dazu die Gründe und den Grad der Verbreitung oder Anerkennung.

Das Fundament aller Artikelarbeit bildet dabei die veröffentlichte, durch eine Peer Group geprüfte Literatur, im Idealfall Fachliteratur. Nur wenn diese zu einem Thema nicht oder noch nicht vorhanden ist, wird auf andere, als verlässlich geltende „Quellen“ zurückgegriffen. Diese „Belegpflicht“ gab es nicht von Anfang an, doch wird sie zunehmend eingefordert. „Artikel sollen nur überprüfbare Informationen aus zuverlässiger Literatur enthalten“ und „In strittigen Fällen können unbelegte Inhalte von jedem Bearbeiter unter Hinweis auf diese Belegpflicht entfernt werden“, wie es in der Wikipedia heißt. ${ }^{7}$ „Die Glaubwürdigkeit der Wikipedia hängt von der Überprüfbarkeit ihrer Inhalte ab.“8

Vor diesem Hintergrund haben es einige angemessen belegte historische Artikel, wie etwa zu römischen Kaisern, zum Frühmittelalter oder zu einer Reihe von mittelalterlichen Königen und Kaisern, aber auch zu Historikern zu einem beachtlichen Niveau gebracht, in dem sich neben dem Lebenslauf und den wis-

7 Vgl. https://de.wikipedia.org/wiki/Wikipedia:Belege (8.3.2015).

8 Vgl. https://de.wikipedia.org/w/index.php?title=Wikipedia:Belege\&oldid=138424587 (8.3. 2015). 
senschaftlichen Schwerpunkten auch der Forschungsstand und die Rezeptionsgeschichte spiegelt. Wir werden hierauf zurückkommen.

\section{Abwehr nicht sinnvoller Eingriffe in Texte und Bilder}

Zunächst jedoch zum weniger erfreulichen Ende der Qualitätsskala. Zwar darf jeder und jede an der Wikipedia mitarbeiten, doch nicht alle Änderungen an den Artikeln sind akzeptabel, viele offenkundig destruktiv, manche gar beleidigend oder völkerverhetzend. Um solcherlei Bekundungen zu unterbinden sind neben einer gewissen Art inhaltlicher Kontrolle einige technische Verfahren entwickelt worden.

In der deutschsprachigen Wikipedia wurde eine Möglichkeit gefunden, unsinnige Änderungen der Texte vorzeitig „abzufangen“, noch bevor sie öffentlich werden. Dies ist für sehr häufig aufgerufene Seiten besonders wichtig, wie etwa für die Hauptseite der Wikipedia, die täglich etwa eine Million Mal aufgerufen wird. ${ }^{9}$ Sie müsste nämlich, selbst wenn nur jeder 10.000ste Besucher auf „Bearbeiten“ klickt, etwas ändert und dann ganz unten auf der Seite auf „Speichern“ klickt und damit „editiert“, zu einer endlosen Kaskade von Neufassungen und demzufolge zu einem Wirrwarr an Änderungen führen (die allesamt in der „Versionsgeschichte“ protokolliert werden). Das geschieht durchaus, doch offenbar nicht so, dass es die Öffentlichkeit zu lesen bekommt. Obwohl es also täglich tausende, nur allzu häufig unsinnige Bearbeitungen an einer Unzahl von Artikeln gibt, erscheinen sie nur selten und fast immer nur für kurze Zeit auf den Bildschirmen der Leser.

Schlichter Vandalismus ist dabei überraschenderweise eines der weniger gravierenden Probleme, mit denen die Macher der Wikipedia täglich zu kämpfen haben. Dies hängt mit einer Art „Filter“ zusammen, der seit einigen Jahren in der deutschsprachigen Wikipedia implementiert ist. Da Menschen, welche die vorhandenen Artikel nicht verbessern, sondern in unsinniger Weise verändern wollen - sie werden innerhalb der Wikipediacommunity als „Vandalen“ bezeichnet -, gern in der Anonymität agieren, klicken sie auf den besagten „Bearbeiten“-Button und speichern damit den Unsinn, den sie in einen der Artikel eingetragen haben.

In ihrer Eile übersehen viele von ihnen beim Speichern offenbar den fett geschriebenen Satz „Deine Änderungen werden angezeigt, sobald sie gesichtet wurden.“ Hinter dem Wort „gesichtet“ verbirgt sich eine Methode, mit der die Änderungen von anonymen „Benutzern“, in diesem Falle eines „Vandalen“, erst

9 Vgl. http://stats.grok.se/de/latest30/Wikipedia:Hauptseite (8.3.2015). 
dann angezeigt werden, wenn ein anderer, der schon ein wenig länger und erkennbar konstruktiv an der Wikipedia mitgearbeitet hat, mit einem Klick zu erkennen gegeben hat, dass die besagte Änderung keinen „Vandalismus“ darstellt. Diese „Wikipedianer“, die sich als produktiv für das Projekt erwiesen haben und von denen es zur Zeit über 15.000 gibt, werden „Sichter“ genannt. ${ }^{10}$ Sie haben bereits eine Mindestzahl an Edits hinter sich, die nicht rückgängig gemacht wurden. Sie haben neben der Sichtung das Recht, mit der Schaltfläche „kommentarlos zurücksetzen“ die letzten Änderungen eines Benutzers mit einem einzigen Klick ohne jedweden Kommentar zu widerrufen und auf die vorhergehende Version zurückzusetzen. Zudem erhalten nur Sichter Einblick in die Liste der noch nicht gesichteten Artikel. ${ }^{11}$ Ihre Tätigkeit beinhaltet also noch keinerlei Qualitätsprüfung oder gar -steigerung, sondern dient nur der Abwehr von offensichtlich destruktivem Verhalten. Erst nachdem einer von diesen Sichtern die besagte Änderung in Augenschein genommen und gewissermaßen genehmigt hat, wird sie überhaupt erst für die anderen Nutzer der Wikipedia sichtbar. Über die inhaltliche oder sprachliche Qualität müssen sie dabei nicht befinden.

Trotzdem kommt es vor, dass auch nicht gesichtete Änderungen zu sehen sind. Dies sollte bei insgesamt über 140 Millionen Seitenbearbeitungen allerdings nicht wundern. ${ }^{12}$ Meist bleibt Vandalismus dementsprechend unsichtbar, genauso wie die Abwehrtätigkeiten anderer Angehöriger der Wikipedia-Community. Bis einer der „Sichter“ die Änderung geprüft hat, kann allerdings einige Zeit vergehen. Damit diese Sichtungen also nicht zu lange auf sich warten lassen, gibt es zum einen regelmäßig aktualisierte Listen der seit längerer Zeit nicht gesichteten Artikel, ${ }^{13}$ zum anderen haben sich knapp 180 „Wikipedianer“ bereiterklärt, solcherlei Nachprüfungen vorzunehmen. Sie sehen ihre Aufgabe also nicht (nur) darin, Artikel zu sichten, sondern vor allem, über längere Zeit nicht gesichtete Artikel „nachzusichten“. ${ }^{14}$

Kehren wir zum Moment des Speicherns zurück. Dem Anonymus, sei er nun guter oder schlechter Absicht, wird zwischen dem Klick auf „Bearbeiten“ und dem auf „Speichern“ ein weiterer Text dargeboten: „Du bearbeitest diese Seite unangemeldet. Wenn du deine Änderung speicherst, wird deine aktuelle IP-Adresse in der Versionsgeschichte aufgezeichnet und ist damit öffentlich einsehbar. Wenn du

10 Vgl. http://de.wikipedia.org/wiki/Spezial:Statistik (8.3.2015).

11 Vgl. http://de.wikipedia.org/wiki/Spezial:Seiten_mit_ungesichteten_Versionen (8.3.2015).

12 Zum aktuellen Stand vgl. http://de.wikipedia.org/wiki/Spezial:Statistik (8.3.2015).

13 Als Beispiel sei hier die Liste der ungesichteten Artikel der Redaktion Antike genannt: http:// de.wikipedia.org/wiki/Wikipedia:Redaktion_Antike/Sichtung (8.3.2015).

14 Vgl. https://de.wikipedia.org/w/index.php?title=Wikipedia:Gesichtete_Versionen/Nachsich tung\&oldid=138841444 (8.3.2015). 
ein Benutzerkonto anlegst, bleibt deine IP-Adresse verborgen. “ Das klingt für viele wie Technik-Chinesisch, doch verbirgt sich Folgendes dahinter: Die IPv4-Adresse, wie sie korrekt heißt, ist eine bis zu 12stellige Zahlenfolge, die jedem Computer zugewiesen wird, der sich in das Internet einwählt - vergleichbar mit einer postalischen Adresse, nur dass sie aus Hexadezimalzahlen besteht. Sie wird an den Server der Wikipedia - den Empfänger - übertragen und beim Speichern des Artikels in der Versionsgeschichte abgespeichert. Sie bleibt dort, solange das Datenbanksystem Wikipedia besteht. Damit werden verschiedene Informationen für den Insider geliefert, darunter der ungefähre Standort des Computers desjenigen Nutzers, der die Specherung vorgenommen hat. Außerdem liefert sie Erkenntnisse darüber, an welchen Stellen der Inhaber dieser IP noch gewirkt hat. Dies ist der Sinn des zunächst rätselhaften Satzes, an dessen Ende eine Alternative angeboten wird, nämlich die Einrichtung eines sogenannten „Benutzerkontos“. Wer also mitarbeiten möchte und Wert auf Anonymität legt - das klingt zunächst paradox -, der oder die sollte sich anmelden.

Man kann bei der Anmeldung einen beliebigen Namen wählen, ein Pseudonym oder den tatsächlichen „Klarnamen“. Wichtigste Bedingung ist, dass sich unter diesem Namen noch kein anderer angemeldet hat, er sollte aber auch keine unverantwortlichen Aussagen enthalten. Danach erst ist es möglich, sich „einzuloggen“, wozu man seinen Benutzernamen und ein frei wählbares Passwort eingibt. Mehr als zwei Millionen solcher Benutzerkonten sind bisher eingerichtet worden, allerdings arbeiten derzeit „nur“ etwa 20.000 dieser Angemeldeten regelmäßig mit.

Doch nicht jeder der über 20.000 aktiven Mitarbeiter ist Sichter oder Autor. Schließlich muss eine Vielzahl von weiteren Aufgaben im Projekt bewältigt werden. Dazu gehört das Erstellen von Karten, Filmen und Fotografien, die Überprüfung von Urheberrechten, aber auch Rechtschreibkorrekturen, Anpassungen an vereinbarte Standards (z. B. folgen alle Texte, die sich auf die Schweiz beziehen der dort gültigen Rechtschreibung), die Einordnung in Kategorien. Dann muss die Technik gewartet und verbessert werden, Fragen von außerhalb wollen beantwortet und Neulinge betreut werden, bis hin zu Pressearbeit und Spendenakquisition (die Wikipedia finanziert sich ausschließlich durch Spenden).

Zudem müssen Meinungsverschiedenheiten geschlichtet und manchmal Nutzer, deren Verhalten sich als untragbar für das Projekt erwiesen hat, ausgesperrt werden. Dazu hat sich die Gemeinschaft der angemeldeten „Wikipedianer“ eine Gruppe von derzeit etwa 250 Menschen per Abstimmung gewählt, die mit besonderen Rechten ausgestattet sind und die zudem Artikel dauerhaft gegen Veränderungen nichtberechtigter Nutzer schützen können, zum Beispiel von nicht angemeldeten „IPs“, die bereits mehrfach Unsinn angerichtet haben, oder von „Projektstörern“. 


\section{Qualitative Verbesserung}

Mit der Anmeldung erhält der Nutzer eine Reihe von neuen Karteireitern am oberen Rand seines Bildschirms. Wo normalerweise nur „Artikel“, „Diskussion“, „Lesen“, „Bearbeiten“ und „Versionsgeschichte“ erscheinen, also die allen unangemeldeten Nutzern zur Verfügung stehenden Mittel der Beteiligung, erscheint als zusätzlicher Button „Beobachten“. Hat man diesen Button betätigt, wird der betreffende, gerade am Bildschirm zu sehende Artikel in die „Beobachtungsliste“ eingefügt. Er kann natürlich auch wieder aus der Liste gelöscht werden („Nicht beobachten").

Oberhalb der besagten Reihe von Karteireitern erscheint darüber hinaus eine zweite Reihe, an deren Anfang der gewählte Benutzername, dann der Link zur eigenen „Diskussionsseite“, die für den Austausch mit anderen Wikipedianern vorgesehen ist. Daneben können die persönlichen technischen „Einstellungen“ den eigenen Bedürfnissen angepasst werden. Schließlich folgen die Karteireiter „Beobachtungsliste“, „Beiträge“ und „Abmelden“. Diese Reihe von Karteireitern erscheint so nur nach erfolgter Anmeldung.

Die Funktion „Beobachtungsliste“ ist für die Beseitigung von Vandalismus und unzutreffenden oder inhaltlich nicht neutralen Beiträgen von größter Wichtigkeit, denn durch Anklicken dieses Buttons werden die Veränderungen an allen Artikeln und Diskussionen aufgeführt, die der angemeldete Nutzer in seine eigene Beobachtungsliste aufgenommen hat. Diese Veränderungen kann man sich durch Vergleich der Versionen im Detail ansehen und, falls nötig, durch einen einfachen Klick wieder rückgängig machen. Unbemerkter Vandalismus wird spätestens hier aufgespürt, da derjenige, der einen Artikel auf seine Beobachtungsliste gesetzt hat, sich aller Wahrscheinlichkeit nach mit dem betreffenden Thema auseinandergesetzt hat, vielleicht sogar von Berufs wegen. So erkennt er auch versteckten Vandalismus, wie die Manipulation von Zahlenangaben. Die Autoren werden ihre eigenen Beiträge sicherlich ganz überwiegend auf ihrer jeweiligen Beobachtungsliste führen. Es ist nicht selten, dass einzelne Nutzer auf diese Weise mehrere tausend Artikel im Blick behalten.

Die meisten Autoren, die sich mit dem Bereich „Geschichte“ befassen, konzentrieren sich auf bestimmte Ausschnitte dieses riesigen Feldes. Die dazugehörigen Kategorien kann man ebenfalls beobachten, so dass neue Einträge gleich bemerkt werden. So können an der Kategorie „Geschichte“ Interessierte die Inhalte überprüfen. Zugleich besteht die Möglichkeit, sogenannte „Fakes“ oder „Hoaxes“ aufzustöbern, also Falschmeldungen, erfundene Artikel, fiktive Biographien, oder als Scherze gemeinte Artikel. Solche Beiträge werden fast ausnahmslos schnell entdeckt und gelöscht. 
Artikel $\mathrm{zu}$ derartigen frei erfundenen Themen oder Personen fliegen gelegentlich auch deshalb auf, weil die angeblichen Publikationen der dargestellten Person im Katalog der Deutschen Nationalbibliothek nicht auffindbar sind. ${ }^{15}$ Der entsprechende Sucheintrag wird von der Artikelseite aus verlinkt, so dass eine Überprüfung leicht möglich ist. Dennoch kann es geschehen, dass in besonders wenig beobachteten und bearbeiteten Bereichen „exotische“ Artikel eine Zeitlang Bestand haben.

Tauchen Artikel auf, deren „Relevanz“ strittig ist, bei denen sich also die Frage stellt, ob sie in die Enzyklopädie aufgenommen werden sollen, kommt es zu einer „Löschdiskussion“ in deren Verlauf ein Abgleich mit den Kriterien für eine ausreichende Bedeutsamkeit erfolgt. In strittigen Fällen entscheiden ein oder mehrere Administratoren.

Schon diese wenigen Worte machen klar, dass das Verfassen und Überprüfen von Artikeln und ihrer Veränderungen nicht so leicht ist, wie man zunächst glaubt. Das wurde nach einigen Jahren auch vielen Beteiligten im Projekt deutlich, so dass im April 2007 ein Verfahren entstand, durch das Neulinge in die technische Seite der Wikipedia, aber auch in die Gepflogenheiten eingeführt werden. ${ }^{16}$ Derzeit gibt es etwa 50 dieser „Mentoren“.${ }^{17}$ Dies ist besonders wichtig, um unnötige Konflikte, etwa um Standards, zu vermeiden.

\section{Anreize zur Qualitätssteigerung}

Um Autoren zur Erstellung qualitativ höherwertige Artikel zu motivieren, sind die besagten Mittel selbstverständlich völlig ungeeignet. Zur Förderung der Motivation gibt es einerseits einen Schreibwettbewerb, der zwei Mal im Jahr ausgetragen wird. Alle Stimmberechtigten, also diejenigen, die konstruktiv mitgearbeitet haben, wählen dazu eine Jury, die sich an einem Ort im deutschen Sprachraum versammelt und sämtliche eingereichten Artikel bewertet, diskutiert und schließlich abstimmt. Seit einigen Jahren gibt es zudem einen Publikumspreis, bei dem jeder mit abstimmen kann.

Auch können Artikel, die für besonders gelungen gehalten werden, jederzeit innerhalb der Wikipedia vorgeschlagen, diskutiert und bewertet werden. Doch nur

15 Hier findet sich ein Beispiel für einen (zumindest bis zum 8. 3. 2015) ungeklärten Fall: https:// de.wikipedia.org/w/index.php?title=Diskussion:Bernhardt_Jungmann\&oldid=75088615 (8.3. 2015).

16 Vgl. https://de.wikipedia.org/wiki/Wikipedia:Mentorenprogramm/R\%C3\%BCckblick (8.3. 2015).

17 Vgl. https://de.wikipedia.org/wiki/Wikipedia:Mentorenprogramm (8.3.2015). 
ein kleiner Teil der derzeit über 1,8 Millionen Artikel (Stand: Ende Januar 2015) gilt dank mittlerweile recht hoher Anforderungen in der Wikipedia als „Lesenswerter Artikel“ oder gar als „Exzellenter Artikel“. Ende Januar 2015 waren es etwas über 6.100 Artikel - also nur jeder dreihundertste -, darunter befanden sich 2.353 exzellente, also solche Beiträge, die in der Wikipedia die höchste Auszeichnung erlangt haben. Diese Artikel sind mit einem grünen Stern rechts oben kenntlich gemacht, die derzeit 3.750 lesenswerten Artikel erhielten einen blauen Stern. Für Listen - etwa die „Liste der Denkmäler in Coburg“, die „Liste der byzantinischen Kaiser“ oder die „Liste der Äbtissinnen von Essen“ - besteht eine eigene Bezeichnung als „informativ“. Auch sie werden mit einem Stern graphisch gekennzeichnet.

Für den Nutzer ist es wichtig zu wissen, dass sich diese Artikel einer Bewertung durch mehrere andere Wikipedianer, meist erfahrene Autoren, unterzogen haben. Der Verlauf der Kandidatur, der sich meist über mehrere Wochen hinzieht, wird nach Abschluss der Kandidatur auf der Diskussionsseite des jeweiligen Artikels abgespeichert und kann daher auch weiterhin nachvollzogen werden. Damit bleiben die Kritikpunkte öffentlich und sind oftmals der Ausgangspunkt für weitere Verbesserungen. In den letzten Jahren sind die Anforderungen an die kandidierenden Artikel erheblich gestiegen, so dass es sich lohnt, das am unteren Ende vermerkte Datum der Auszeichnung zu prüfen, aber auch, sich die jüngeren Einträge auf der Diskussionsseite anzusehen - sie befinden sich ganz unten auf der Seite.

Neben der alphabetischen Liste aller ausgezeichneten Artikel sind auch alle Artikel aus dem Bereich der Geschichte zu finden. ${ }^{18}$ Darunter befinden sich in den jeweils geringfügig voneinander abweichenden Themenbereichen folgende Artikel (s. Tabelle S. 194).

Insgesamt sind etwa 600 historische Artikel „lesenswert“, etwa 440 „exzellent“, so dass man sagen kann, die Geschichte hat in den letzten Jahren gegenüber den Naturwissenschaften stark aufgeholt. Sie stellt inzwischen ein Drittel der ausgezeichneten Artikel. Hinzu kommen 58 sogenannte „informative Listen“ ${ }^{19}$, darunter befinden sich die Äbtissinnen von Essen oder die Bischöfe von Regensburg, eine Liste der byzantinischen und eine der römischen Kaiser, aber auch eine „Liste der Kaiser der Jin-Dynastie (265-420)“, dann eine der Dogen von Venedig oder der Sultane des Osmanischen Reiches, der Maya-Ruinen, aber

18 Vgl. Wikipedia:Exzellente Artikel/Geschichte: http://de.wikipedia.org/wiki/Wikipedia:Exzel lente_Artikel/Geschichte (Wikipedia:Exzellente Artikel/Geschichte) (eingesehen am 8.3.2015). 19 Vgl. Informative Listen und Portale: http://de.wikipedia.org/wiki/Wikipedia:Informative_Lis ten_und_Portale (8.3.2015). 
ebenso eine mit dem Titel „Marschälle der Sowjetunion“ oder gar die „Top-30Schellackplatten des Duke Ellington Orchestra“.

Tabelle: Artikel zu historischen Themen mit ausgezeichneter Qualität (Stand: Januar 2015).

\begin{tabular}{lrr}
\hline Themenbereich & lesenswert & exzellent \\
\hline Ur- und Frühgeschichte, Ägyptologie und Altorientalistik & 61 & 37 \\
Antike & 53 & 79 \\
Frühzeit außerhalb Europas und Vorderasiens & 10 & 4 \\
Mittelalter & 67 & 52 \\
Frühe Neuzeit & 55 & $45^{20}$ \\
\hline 18. Jahrhundert & $32^{21}$ & - \\
19. Jahrhundert & 64 & 60 \\
Imperialismus & 20 & - \\
1900-1933 & - & 41 \\
Erster Weltkrieg & 10 & 0 \\
\hline Zwischenkriegszeit & 25 & 0 \\
Zeit des Nationalsozialismus und & 80 & 65 \\
Zweiter Weltkrieg (1933-1945) & & \\
seit 1945 bis heute & 62 & 30 \\
historische Orte und Gebäude & 39 & 21 \\
Militärgeschichte & 9 & 3 \\
\hline
\end{tabular}

Ein weiteres Mittel zur Qualitätsverbesserung sollte die Auslobung der JohannHeinrich-Zedler-Medaille sein. Deren Vergabe wird seit 2007 von Wikimedia Deutschland e.V. ausgelobt, einem Förderverein für die Wikipedia, der jedoch keine Mutterstiftung darstellt. Dies geschieht in Zusammenarbeit mit Spektrum der Wissenschaft und der Akademie der Wissenschaften und der Literatur Mainz in den beiden Kategorien Geistes- bzw. Naturwissenschaften. Zur Jury gehörten Historiker wie Johannes Fried. 2010 erweiterten die Träger den Preis um einen Bilderwettbewerb und erhielten außerdem Unterstützung von BASF, Carl Zeiss sowie Reporter ohne Grenzen. Ab 2012 wurde die Medaille vom Zedler-Preis für Freies Wissen abgelöst. Auch im weiteren Sinne historische Projekte und auch einzelne Artikel wurden damit ausgezeichnet, wie das Österreichische Portal Denkmallisten, dann 2013 das „WikiProjekt Osttimor“ oder der Artikel „El Greco“.22

20 Bei den sogenannten Exzellenten Artikeln offenbar weiter gefasst.

21 Nur bei den sogenannten Lesenswerten Artikeln.

22 Vgl. https://www.wikimedia.de/wiki/Zedler-Preis (8.3.2015). 
Ende 2013 wurde das Förderprogramm Freies Wissen durch Wikimedia Deutschland eingeführt. Es soll Ideen aus der Wikipedia selbst fördern, der sogenannten Community. ${ }^{23}$

Nicht nur bei Texten, sondern auch bei Bild- und Tonmedien stellt sich die Frage nach der Qualität und darüber hinaus der Verlässlichkeit der Beschreibungen. Wikimedia Commons bietet für die in verschiedenen Sprachen entstandenen „Wikis“ inzwischen fast 25 Millionen Dateien an. ${ }^{24}$ Dabei handelt es sich hauptsächlich um Bilddokumente, aber auch Ton- und Filmaufnahmen. Zu diesem Bestand trugen neben Einzelinitiativen und Millionen privater Bildspenden vor allem Archive bei, wie 2008 das Bundesarchiv durch eine große Bilderspende von über 80.000 Bildern. ${ }^{25} 2009$ folgte die Deutsche Fotothek $(250.000)^{26}$ und das Königliche Niederländische Tropeninstitut (über 49.000), 272010 das niederländische Nationaal Archief (über 13.000). ${ }^{28}$ Damit wurden nicht nur wichtige historische Bilder für die Verwertung durch Wikipedia freigegeben, sondern auch von verlässlichen Institutionen öffentlicher Hand beschriebene Medien überreicht, was die größtmögliche Sicherheit der Zuordnung und Beschreibung, aber auch vice versa gewährleistet. Auch Google Art stellt zahlreiche Abbildungen aus dem Bereich der Kunst zur Verfügung. Diese Bilder kann man nach Künstlernamen durchsuchen. ${ }^{29}$

Zugleich wurden die Medien einem Kategoriebaum zugeordnet, so dass die Möglichkeiten, zum eigenen Text passende Medien zu finden, enorm verbessert wurden. Wer sich durch diesen inzwischen stark verästelten Kategorienbaum „durchhangelt“, der wird für seine Fragestellungen immer häufiger fündig.

Hinter den Artikeln steckt eine Menge Arbeit, zumal die wesentlichen oder umstrittenen Aussagen in Form von Fußnoten seit 2005 belegt sein sollen. Dies

23 Vgl. https://de.wikipedia.org/w/index.php?title=Wikipedia:F\%C3\%B6rderprogramm_Frei es_Wissen\&oldid=139521722 (8.3.2015).

24 Vgl. https://commons.wikimedia.org/w/index.php?title=Hauptseite\&oldid=151977170 (8. 3. 2015).

25 Vgl. https://commons.wikimedia.org/w/index.php?title=Commons:Bundesarchiv\&oldid=135 426501 (8. 3. 2015).Vgl. Sander, Oliver: „Der Bund mit Wiki“ - Erfahrungen aus der Kooperation zwischen dem Bundesarchiv und Wikimedia, in: Der Archivar 63 (2010), H. 2, S. 158-162. 26 Vgl. https://commons.wikimedia.org/w/index.php?title=Commons:Deutsche_Fotothe k\&oldid=135427610 (8.3.2015).

27 Vgl. https://commons.wikimedia.org/wiki/Category:Images_from_the_Tropenmuseum (8.3. 2015).

28 Vgl. https://commons.wikimedia.org/w/index.php?title=Category:Images_from_Nationaal_ Archief\&oldid=145875269 (8.3.2015).

29 Vgl. https://commons.wikimedia.org/wiki/Category:Google_Art_Project_works_by_artist (8.3. 2015). 
war in den ersten Jahren der Wikipedia nicht der Fall. Eine Belegpflicht für alle neuen Artikel wurde mit $55 \mathrm{zu} 111$ Stimmen im September 2006 abgelehnt. $^{30}$

Bei der Markierung von Belegmängeln geht die deutschsprachige Wikipedia eigene Wege. So lehnte sie im Februar 2013 mit über 70 Prozent der Abstimmenden den Vorschlag ab, in den jeweiligen Artikel an allen Stellen, an denen ein Beleg erforderlich sein könnte, einen Hinweis („citation needed“) einzufügen. ${ }^{31}$ Die Erfahrung in der englischsprachigen Version der Wikipedia zeigt, dass etwa die Hälfte der entsprechenden Markierungen sich auf diesen Mangel bezieht. Insgesamt beziehen sich fast zwei Drittel dieser Markierungen auf Qualitätsmängel. ${ }^{32}$ Dabei waren 2013 fast 27 Prozent mit mindestens einem der 445 sogenannten tags versehen, die Qualitätsmängel anzeigen sollen. Mehr als drei Viertel bezogen sich auf den gesamten Artikel, der Rest auf einen bestimmten Teil. 50 Prozent bezogen sich dabei auf die Belegsituation. ${ }^{33}$

Kritisiert wurde, dass in der englischsprachigen Wikipedia über 250.000 Artikel mit ${ }^{\text {[citation needed] }}$ versehen worden seien. Außerdem seien sie zu unspezifisch, würden kaum zu Überarbeitungen anregen, vielfach willkürlich „verteilt“. Zudem verschlechterten sie die Lesbarkeit, seien ungünstig für die Barrierefreiheit, führten zu ausufernden Diskussionen.

Bei den „Belegen“ gilt es einige Spezifika der Wikipedia zu beachten. Die Belege dürfen nur in Ausnahmefällen Quellen im Sinne der historischen Wissenschaften sein. Sie dürfen sich also nur auf die Fachliteratur oder andere verlässliche Publikationen beziehen, nicht, um ein Beispiel $\mathrm{zu}$ nennen, auf eine mittelalterliche Urkunde. Dies ist ein wesentliches Unterscheidungsmerkmal zu Artikeln in Fachzeitschriften, die neben der Fachliteratur selbstverständlich auf Quellen zurückgreifen müssen. Diese Angaben findet man in den Geschichtswissenschaften im Allgemeinen unter „Anmerkungen“ - wobei in der Wikipedia eine gewisse Eigendynamik in der sprachlichen Gestaltung auftreten kann, was zu absonderlichen Wortbildungen wie „Einzelbelege“34 geführt hat. Es ist aber typisch für ein so großes Projekt, dass die Rückbindung an die wissenschaftlichen Traditionen punktuell und zeitweise verloren gehen kann. Mitunter ist dies auch technischen Experimenten aus der Programmiererwelt geschuldet, doch diese

30 Vgl. https://de.wikipedia.org/wiki/Wikipedia:Meinungsbilder/Quellenpflicht_f\% $\%$ C3 \%BCr_ neue_Artikel\#Problematik (8.3. 2015).

31 Vgl. https://de.wikipedia.org/w/index.php?title=Wikipedia:Meinungsbilder/Vorlage_zur_Mar kierung_von_Belegm\%C3 \%A4ngeln\&oldid=124200087 (8.3.2015).

32 Anderka, Maik: Analyzing and Predicting Quality Flaws in User-generated Content: The Case of Wikipedia, Dissertation, Bauhaus-Universität Weimar 2013, S. 85.

33 Ebd., S. 84.

34 Diese Bezeichnung findet sich in etwa 4.100 Artikeln. 
können so gut wie nie gegen die über Jahrhunderte entwickelte Logik des wissenschaftlichen Apparats dauerhaft bestehen. $\mathrm{Zu}$ widersprüchlich und unklar abzugrenzen sind die Ergebnisse, was wiederum zu zahllosen Diskussionen und Streitigkeiten führte. So wurden Versuche unternommen, „Belege“ anhand der Literatur und „Anmerkungen“ im Sinne von Erläuterungen zu trennen. Vielfach führte auch der für die historischen Wissenschaften spezifische Begriff der Quelle, die ja eine in der Wikipedia nicht gewünschte Quellenkritik erfordern würde, in der Auseinandersetzung mit anderen Wissenschaften zu Missverständnissen.

Um Neueinsteigern den Einstieg in die Codierung der Belege zu erleichtern, sollte diese möglichst einfach gehalten werden, doch ist die Diskussion darüber noch im Fluss.

Um aktiv, also nicht im Sinne einer Abwehr von Verschlechterungen, Qualitätsverbesserungen $\mathrm{zu}$ erreichen, geht die Wikipedia einen weiteren Weg. Im Bereich der Geschichte hat sich, wie in einigen anderen Fächern auch, die Einrichtung einer Gruppe von Fachleuten durchgesetzt. Bei den Historikern ist dies die „Redaktion Geschichte“, für die Antike existiert eine „Redaktion Antike“, ebenso wie für zahlreiche Themen, wie das Militärwesen oder die Vereinigten Staaten, die auch immer historische Anteile aufweisen. Die Redaktion Geschichte besteht seit dem 25. März 2006. ${ }^{35}$ Dort kann sich jeder mit seinen inhaltlichen Schwerpunkten eintragen, der an der Arbeit der Redaktion teilnehmen will. Die Redaktionsseite wird durchschnittlich mehr als 100 Mal pro Tag aufgerufen. ${ }^{36}$

Problematische Artikel werden dort in einer eigenen Beobachtungsliste geführt, externe Webseiten besprochen oder neue Webseiten bewertet, Artikel überarbeitet oder auf Projekte oder neue Forschungsergebnisse hingewiesen. Auch steht eine Seite der Redaktion zum Bereich „Hilfsmittel für Autoren“ zur Verfügung. ${ }^{37}$ Hinzu kommt die Qualitätssicherungsseite, ${ }^{38}$ wo mangelhafte Artikel eingetragen werden können, um eine Überarbeitung zu veranlassen. Auch dies geschieht wiederum auf freiwilliger Basis, wobei immer wieder mehrere Autoren, die in bestimmten Feldern beschlagen sind, zusammenarbeiten. Einige Autoren geben ihre akademische Grade bekannt, doch viele von ihnen wollen anonym bleiben. Dies ist in historischen Feldern, die als brisant gelten, eine gut vertretbare

35 Vgl. https://de.wikipedia.org/w/index.php?title=Wikipedia:Redaktion_Geschichte\&oldid=14 998725 (8.3.2015).

36 Vgl. die Aufrufe der letzten 90 Tage: http://stats.grok.se/de/latest90/Wikipedia:Redaktion_Ge schichte (8.3.2015).

37 Vgl.https://de.wikipedia.org/wiki/Wikipedia:Redaktion_Geschichte/Hilfsmittel_f\%C3\%BCr_ Autoren (8.3.2015).

38 Vgl. https://de.wikipedia.org/wiki/Wikipedia:Redaktion_Geschichte/Qualit\%C3\%A4tssiche rung (8.3.2015). 
Entscheidung. Auch möchten manche Autoren nicht mit der Wikipedia als Wissenschaftler in Verbindung gebracht werden. Andere wiederum nutzen die Gelegenheit, sich an „Stammtischen“ - einen Historikerstammtisch gibt es allerdings nicht -, die es in einer ganzen Reihe von Städten gibt, regelmäßig zu treffen und auszutauschen. ${ }^{39}$

Neben den Redaktionen ist eine Reihe von Portalen entstanden, die sich historischen Themenkreisen widmen. ${ }^{40}$ Sie sollen einen größeren Themenkomplex systematisch erschließen. Der Fokus liegt darin, den Lesern die Inhalte ihrer Themenbereiche strukturiert darzustellen. So bestehen Portale zu den Themenkreisen Ur- und Frühgeschichte sowie Archäologie, Ägyptologie und Alter Orient, Altertum, Griechische Antike und Rom, Byzanz, Mittelalter und Heiliges Römisches Reich, Frühe Neuzeit, Preußen und Ostpreußen, 19. Jahrhundert, 20. Jahrhundert, Imperialismus und Weltkriege, Britisches Weltreich, Nationalsozialismus und DDR, Sowjetunion, aber auch Wilder Westen, Sezessionskrieg und Pazifikkrieg, schließlich Biografien und Numismatik. ${ }^{41}$

Neben diesen Portalen bestehen Einzelprojekte, überwiegend als Projekte der jeweiligen Portale, zur Vor- und Frühgeschichte, zur Ägyptologie, zu den Germanen sowie zum Römischen Limes und zum Römischen Konsulat. Zum Mittelalter besteht derzeit kein Projekt, jedoch eines zur Frühen Neuzeit, dann zum Pazifikkrieg und zu Imperialismus und Weltkriege, schließlich zur Geschichte Russlands und zum 19. Jahrhundert. ${ }^{42}$ Ähnlich wie die Redaktionen dienen sie als Platz für die inhaltliche Arbeit, themenspezifische Kommunikation, aber auch zur Quellensammlung und Zusammenfassung von Hilfestellungen. Sie richten sich gezielt an Autoren und versuchen Autoren zu gewinnen.

39 Eine Übersicht über die Stammtische findet sich hier: https://de.wikipedia.org/wiki/Wikipe dia:Treffen_der_Wikipedianer (8.3.2015).

40 Hier findet sich eine Liste der historischen Portale: https://de.wikipedia.org/wiki/Kategorie: Portal:Geschichte_als_Thema (8.3.2015).

41 Vgl. https://de.wikipedia.org/wiki/Portal:Mittelalter, 8.3.2015, mit der Verlinkung zu den genannten Portalen. Vgl. auch https://de.wikipedia.org/wiki/Wikipedia:WikiProjekt_Pazifikkrieg (8.3.2015).

42 Vgl. https://de.wikipedia.org/wiki/Wikipedia:WikiProjekt (8.3.2015). 


\section{Sonderbefugnisse zur Konfliktlösung, Abstimmungen und Befragungen}

Wie schon zu Anfang erwähnt, kommt es durch sogenannte „Vandalen“ vielfach zu destruktiven Eingriffen gewissermaßen von außen. Doch auch innerhalb der Autorenschaft kommt es zu Konflikten und Regelverstößen.

Zur Lösung solcher Konflikte zwischen Autoren oder Benutzern wurde am 27. April 2007 ein Schiedsgericht eingeführt. Derzeit gehören dem Schiedsgericht zehn Mitglieder an. Sie werden in jährlich zwei Wahlen jeweils für ein Jahr gewählt. Dabei ist die Wiederwahl möglich. Die Kandidaten müssen mindestens 400 akzeptierte Bearbeitungen gespeichert haben, zudem stimmberechtigt sein - allein dazu muss der Kandidat mindestens 200 Bearbeitungen an Artikeln vorgenommen haben, davon mindestens 50 in den letzten zwölf Monaten - und seit mindestens vier Monaten vor der Wahl in der Wikipedia aktiv gewesen sein. Mitglieder des Schiedsgerichts erhalten auch die Rechte der „Administratoren“.

Mitarbeiter an der Wikipedia können sich durch eine Kandidatur von der gesamten deutschsprachigen Community zu einem der momentan etwa 250 Administratoren wählen lassen. Bei der Kandidatur ist ein Mindesquorum von zwei Dritteln der abstimmungsberechtigten „Wikipedianer“ vonnöten. Dabei müssen innerhalb von zwei Wochen mindestens 50 Stimmberechtigte mit Pro abgestimmt haben. Zwar dürfen sie kurze Begründungen einfügen, jedoch nicht die Begründungen anderer kommentieren. ${ }^{43}$

Den Administratoren obliegen viele klassische Verwaltungsaufgaben. Dabei handelt es sich um stimmberechtigte und angemeldete Benutzer, denen das Vertrauen entgegengebracht wird, mit ihren zusätzlichen Werkzeugen im Sinne der allgemein anerkannten Grundsätze der Wikipedia zu handeln und dabei ihre eigenen Interessen und Standpunkte zurückzustellen. Sie sind in besonderem Maße auf überlegtes Handeln und Vorbildfunktion festgelegt. Analog zur „Netiquette“, in der Kriterien für das Verhalten im Internet aufgestellt wurden, entstand die „Wikiquette“ für das angemessene Verhalten im Rahmen der Wikipedia, also Grundsätze, die für Administratoren in besonderem Maße gelten. Die Administratorenrechte werden Benutzern verliehen, die demnach in einem weiteren Sinne bereits eine längere Zeit konstruktiv zur Wikipedia beigetragen haben. Sie tragen jedoch keine Verantwortung für die Inhalte der Artikel. Verstößt ein Administrator gegen zentrale Grundsätze des Projektes, kann er abgewählt werden.

43 Vgl. die Wahlseite: https://de.wikipedia.org/wiki/Wikipedia:Kandidaturen\#Adminkandidatu ren (8.3.2015). 
Dazu muss er sich erneut den stimmberechtigten „Wikipedianern“ zur Wahl stellen.

$\mathrm{Zu}$ den Rechten der Administratoren gehört das Löschen und Sperren von Seiten, aber auch das Sperren von angemeldeten Benutzern, die sich trotz mehrfacher Ermahnung (bei besonders schwerwiegenden Fällen ohne Vorwarnung), nicht an die Regeln halten. Benutzerkonten können allerdings nur bei gravierenden Regelverstößen oder Missbrauch des Accounts gesperrt werden, etwa, weil sich bei einer Überprüfung herausgestellt hat, dass zum Zweck der Manipulation von Abstimmungen oder Wettbewerben mehrere Accounts eingerichtet worden sind. Erst recht gilt dies für Belästigungen anderer Nutzer oder gar strafbare Handlungen oder Aufrufe dazu.

Neben der technischen Berechtigung treten Administratoren auch als entscheidende Instanz auf verschiedenen Projektseiten wie den Diskussionen über die Löschung von Artikeln oder bei der sogenannten „Vandalismusmeldung“ auf, wo Regelverstöße und deren Verursacher aufgeführt, diskutiert und gegebenenfalls geahndet werden. Falls sich verschiedene Autoren nicht einigen können, kann ein Administrator eine Seite auch „schützen“, das heißt, sie gegen weitere Veränderungen für eine gewisse Zeit oder dauerhaft sperren. Diese Sperren müssen durch den Administrator begründet werden. Sie erscheinen in einem eigenen Sperrlogbuch.$^{44}$ Artikel, die besonders umstritten sind, finden sich auf einer entsprechenden Liste nach übergreifenden Themen geordnet. ${ }^{45}$

Da alte Versionen über die Versionsgeschichte weiterhin erreichbar bleiben, wurden Urheberrechtsverletzungen oder illegale Inhalte, persönliche Diffamierung oder die Offenlegung privater Informationen ein Problem, da diese auch nach Löschung im Artikel selbst durch Konsultation der Versionsgeschichte noch abgerufen werden konnten. Administratoren können daher einzelne Artikelversionen nachträglich ,verstecken“. Sie sind damit nicht wirklich gelöscht, sind jedoch nur noch für die Administratoren einsehbar (und wiederherstellbar). Sie erscheinen erst recht nicht bei den Suchergebnissen von Google und sind auch über einen Permalink nicht mehr auffindbar. Landet man durch Anklicken eines Permalinks, der auf eine solche gelöschte Version verweist, so erhält ein Administrator die Meldung „Diese Version wurde gelöscht und ist nicht mehr öffentlich einsehbar. Nähere Angaben zum Löschvorgang sowie eine Begründung finden sich im „Lösch-Logbuch“. Jeder Benutzer, der mit weniger Rechten ausgestattet ist,

44 Vgl. das Seitenschutz-Logbuch: https://de.wikipedia.org/wiki/Spezial:Logbuch/protect (8.3. 2015).

45 Zu den Beobachtungskandidaten vgl. https://de.wikipedia.org/wiki/Wikipedia:Beobachtungs kandidaten (8.3.2015). 
erhält die Versionsgeschichte, in der die gelöschte Version grau dargestellt und nicht mehr anklickbar ist. Damit ist sie öffentlich nicht mehr einsehbar.

Um eine solche Version endgültig zu löschen, also auch vor den Augen der Administratoren zu verbergen, gibt es das Amt des „Oversighters“. Hat dieser einen Eintrag gelöscht kann er auch von den Administratoren nicht mehr eingesehen werden und er ist nur noch Oversightern zugänglich. Solche Entfernungen und Löschungen werden vor allem dann vorgenommen, wenn es sich um schwerwiegende persönliche Angriffe handelt, um Verleumdungen, Kriminelles, die Entfernung von nicht-öffentlichen persönlichen Informationen - auch solchen, die der Betreffende explizit als privat bezeichnet hat - sowie bei Urheberrechtsverletzungen. Oversighter müssen bereit sein, ihre Identität gegenüber der Wikimedia-Foundation offenzulegen. Sie betreibt die Server auf denen die Wikipedia-Daten gehostet werden und deren Verwaltung.

Im Laufe der Zeit werden immer wieder Sonderfunktionen durch Wahl besetzt, um ganz spezifische Aufgaben zu lösen. Die fünf von der Community gewählten „Bürokraten“ der deutschsprachigen Wikipedia haben einerseits die technische Möglichkeit, Benutzern die besagten Administratoren-, oder Bürokratenrechte einzuräumen. Auch hier geht die Wikipedia des deutschen Sprachraums einen eigenen Weg, denn in anderen Wikimedia-Projekten haben sie hierin keine Rechte. Ein Benutzer wird zum anderen durch die erfolgreiche Kandidatur zwar automatisch zum Administrator, doch bei einem sehr knappen Ergebnis kann der Bürokrat die Rechtevergabe verweigern. ${ }^{46}$

Zum Entzug der Rechte sind nur Stewards berechtigt; sie haben auch Oversight-Zugang, haben also die noch einmal deutlich erweiterten Rechte dieser oben bereits erwähnten Gruppe. Doch darüber hinaus sind sie international tätig, das heißt sie können auch in anderen Sprachversionen der Wikipedia eingreifen.

Eine unter dem Aspekt des Datenschutzes besonders verantwortungsvolle Tätigkeit stellt der „Checkuser“ oder die „Check User-Abfrage“ dar. Diese Abfrage kann bei Verdacht auf erheblichen Missbrauch der Wikipedia zum Einsatz kommen. Unter Berücksichtigung von thematischen, inhaltlichen, sprachlichen, syntaktischen, technischen, zeitlichen und anderen Eigenheiten der Bearbeitungen lässt sich eine häufig an Sicherheit grenzende Wahrscheinlichkeit ermitteln, dass Bearbeitungen vorgeblich verschiedener Benutzer tatsächlich von derselben Person vorgenommen wurden. Besitzt diese Person mehrere Accounts oder arbeitet anonym, nutzt sie also durch Vortäuschen unterschiedlicher Identitäten die Möglichkeit zur Umgehung einer Sperre, zur Verfälschung von Wahlen

46 Vgl. zu den Bürokratenwahlen: https://de.wikipedia.org/wiki/Wikipedia:B\%C3\%BCrokraten kandidaturen/Archiv (8.3.2015). 
durch mehrfache Stimmabgabe oder zur Manipulation von Diskussionen durch Vortäuschen von Mehrheiten, so kann der Nutzer gesperrt werden - eventuell mitsamt seinen Nebenaccounts, die als „Sockenpuppen“ bezeichnet werden eine etwas unglückliche Übernahme aus dem Angelsächsischen sock puppetry. Bei all diesen Untersuchungen sind strenge Datenschutzgrundsätze zu beachten und die Persönlichkeitsrechte zu berücksichtigen.

Als „Office Actions“ werden Eingriffe der Wikimedia Foundation bezeichnet, dem Dienstanbieter der deutschsprachigen Wikipedia. Sie werden dann durchgeführt, wenn es um Verleumdungen, den Schutz der Privatsphäre oder die Verletzung von Urheberrechten geht. Grundsätzlich handelt es sich dabei um juristisch relevante Verstöße.

Nach demokratischen Grundsätzen werden grundsätzliche, nicht juristisch bedeutsame Fragen geklärt. Dazu dienen „Meinungsbilder“. Meinungsbilder dienen der Klärung besagter Fragen und der Herstellung von Konsistenz, nicht der Klärung inhaltlicher Divergenzen oder gar Auseinandersetzungen um einzelne Artikel. ${ }^{47}$ Ziel ist es, eine für möglichst viele Benutzer akzeptable und praktikable Richtlinie zu finden, etwa um die Erweiterung der Rechte von Sichtern. ${ }^{48}$ Sie werden abgehalten, wenn sich keine Einigung erzielen lässt. Auch hier haben sich im Laufe der Jahre Regularien entwickelt.

Weniger formell sind Umfragen, deren Ergebnisse vielfach über Meinungsbilder zu grundsätzlichen Entscheidungen führen können. Solche Meinungsbilder und Umfragen können jederzeit von jedermann initiiert werden, doch sollten ihre Zahl gering bleiben und bestimmte Regularien beachtet werden. ${ }^{49}$

\section{Hauptautor, Zitiermöglichkeiten}

Bei häufig veränderten Artikeln ist es schwierig herauszufinden, wer den überwiegenden Anteil an der Arbeit geleistet hat. Dies mag neben der Sorge, dass jeder in seinem Werk „herumpfuschen“ kann, den ein oder anderen von der Mitarbeit abschrecken. Doch gibt es inzwischen Werkzeuge, mit denen der Anteil der jeweiligen Nutzer recht genau herausgefiltert werden kann. ${ }^{50}$

47 Vgl. https://de.wikipedia.org/wiki/Wikipedia:Meinungsbilder (8.3.2015).

48 Vgl.https://de.wikipedia.org/wiki/Wikipedia:Meinungsbilder/Erweiterung_der_Sichterrechte (8.3.2015).

49 Näheres findet sich hier: https://de.wikipedia.org/wiki/Wikipedia:Meinungsbilder (8.3.2015).

50 So findet man für den Artikel „Republik Venedig“ ein entsprechendes Werkzeug hier: https:// tools.wmflabs.org/xtools/articleinfo/index.php?pageid=98407\&project=de.wikipedia.org\&use 
Was die Motivation manches Historikers lähmt, an der Wikipedia mitzuarbeiten, ist die Tatsache, dass es bisher kaum möglich ist, einen solchen Beitrag zu zitieren und in den fachlichen Diskurs einzuordnen. Doch auch hier gibt es inzwischen Ansätze seitens der Wikipedia, ${ }^{51}$ ebenso wie von der Seite der Historiker her (s. Beitrag Thomas Wozniak in diesem Band), denen bisher als erster Ansatz nur die Möglichkeit blieb, sich mit dem sogenannten „Klarnamen“ anzumelden. Doch dem Außenstehenden ist selbst dann nicht sofort klar, dass sich hinter dem Klarnamen tatsächlich der gleichnamige Historiker verbirgt - oder besser: zeigt.

Angesichts des geringen Alters der Internetenzyklopädie mag es noch nicht von besonderer Bedeutung sein, sie selbst als historische Quelle zu nutzen, doch hat sich dies in einigen Bereichen bereits begonnen zu ändern. Mit Blick auf die Zukunft könnte dabei die Möglichkeit, die Wikipedia zu zitieren, aus der Enzyklopädie eine Quelle machen, wie wir es von den älteren Enzyklopädien, die für zahlreiche Fragestellungen herangezogen wurden, auch der Fall ist. Dazu müsste, auch dies ist technisch möglich, auf die Version des zu zitierenden Artikels zu einem bestimmten Zeitpunkt verwiesen werden. Nur diese älteren Versionen sind stabil, der eigentliche Artikel wird immer wieder verändert und mit einiger Wahrscheinlichkeit dabei verbessert.

\section{Beraten, einmischen, einüben - weil jeder mitschreiben darf}

Selbstverständlich können hier nur die wichtigsten Mittel und Wege aufgeführt werden, auf denen formell und informell Qualität und Reichweite der Wikipedia durch zuweilen unglaublich fleißige Hände verbessert werden. Was treibt diese Menschen an?

Nach meiner inzwischen mehr als achtjährigen Erfahrung als Autor und fünfjährigen als „Administrator“, ist es sehr häufig gesellschaftliche Verantwortung. Wer außer ausgebildeten Historikern, aber auch gut eingearbeiteten Laien, soll denn an einer gesellschaftlich so einflussreichen Stelle, wie sie die Wikipedia darstellt, all das verhindern, was man mit Geschichte anstellen kann und bereits angestellt hat? Jeder, der gesellschaftliche Verantwortung als historisch gebildeter Mensch zu übernehmen bereit ist, sollte das Feld, das er oder sie beherrscht, nicht

lang=de, für Kaiser Friedrich III. hier: https://tools.wmflabs.org/xtools/articleinfo/index.php?pag eid=28911\&project=de.wikipedia.org\&uselang=de (8.3.2015).

51 https://de.wikipedia.org/w/index.php?title=Wikipedia:Hauptautoren\&oldid=139368649 (8.3.2015). 
nur in Fachzeitschriften, sondern auch in der Wikipedia mit ihrer großen Reichweite keinesfalls anderen überlassen. Beiträge von Fachleuten zu deren Themenkreisen sind daher besonders willkommen. Offenkundige Fehler auf Diskussionsseiten anzumerken wäre bereits ein wichtiger Schritt zur qualitativen Verbesserung - nach der Kapitulation des Brockhaus nunmehr der einzigen allgemeinen Enzyklopädie neben der Encyclopedia Britannica, die aber, so die Ankündigung von 2012,52 auch nur noch digital erscheinen wird. Oder wie Peter Burke 2012 den zweiten Band seiner Social History of Knowledge so pointiert betitelte: From the Encyclopaedia to Wikipedia. ${ }^{53}$

Vielleicht gibt es aber auch einen ganz pragmatischen Grund, warum Lehrende ihre Studierenden zur Partizipation an der Wikipedia ermuntern sollten. Eine australische Studie konnte zeigen, dass sich die Fähigkeiten, die zum Abfassen von Texten erforderlich sind, beim Schreiben für ein tatsächlich existierendes Publikum erheblich besser entwickelten, als wenn die Studierenden nur für ihre Lehrenden geschrieben hätten. ${ }^{54}$ Ähnliches gilt für andere wichtige Fertigkeiten, die die Studierenden für eine akademische Laufbahn brauchen. ${ }^{55}$ Einen Text unter den kritischen Augen einer mitlesenden Öffentlichkeit abzufassen, ist keine leichte, aber äußerst inspirierende Aufgabe. Die meisten entwickeln dort überhaupt zum ersten Mal einen Gedanken in einem Text und grabschen nicht gleich nach dem Ergebnis. Wer dann auch noch Vorbilder sieht und Hilfe annehmen kann, der ist - sieht man von der eigentlichen Forschungsarbeit inklusive der Verarbeitung von Quellen ab - bestens vorbereitet für kollaboratives Arbeiten und zugleich produktive Einsamkeit, zumindest aber für das Abfassen von allem zwischen Hausarbeit und akademischer Abschlussarbeit. In jedem Falle kann die Fähigkeit eingeübt werden, den Forschungsstand zu erarbeiten und angemessen darzustellen.

52 After 244 Years, Encyclopaedia Britannica Stops the Presses, in: Media Decoder vom 13. März 2012. Vgl. http://mediadecoder.blogs.nytimes.com//2012/03/13/after-244-years-encyclopaediabritannica-stops-the-presses/ (8.3.2015).

53 Burke, Peter: Social History of Knowledge, Bd. 2: From the Encyclopaedia to Wikipedia. Cambridge 2012.

54 Miller, Julia: Building academic literacy and research skills by contributing to Wikipedia: A case study at an Australian university, in: Journal of Academic Language and Learning 8,2 (2014), S. A72-A86.

55 Schwerte, Thorsten; Stolz, Stefan: Preparing and publishing Wikipedia articles are a good tool to train project management, teamwork and peer reviewed publishing processes in life sciences, in: Improving University Teaching. The University of British Columbia, 2012. http://www.iutconfer ence.com/2014/03/preparing-and-publishing-wikipedia-articles-as-training-tool-in-project-ma nagement-teamwork-and-the-peer-review-publishing-process-in-the-life-sciences/ (7.3.2015). 